\title{
Searches for Dark Matter with the IceCube Neutrino Telescope
}

\author{
Sebastian Baur, for the IceCube collaboration* \\ Université Libre de Bruxelles, Brussels, Belgium
}

\begin{abstract}
The nature of dark matter is one of the long-standing open questions in modern cosmology. While many different experimental methods are being explored, a clear signature for particle dark matter is yet to be found. In indirect searches, the final state particles of decaying or self-annihilating dark matter could be observed with existing astro-particle experiments. Due to their small cross-section, neutrinos are able to escape from dense environments such as the Sun or the Earth which makes them unique messengers for dark matter searches. The IceCube neutrino telescope has a diverse program on dark matter searches exploring different source regions and possible mass-ranges. Furthermore, various models such as decaying, annihilating or secluded dark matter are studied.
\end{abstract}

Keywords: dark matter, indirect searches, neutrinos, IceCube

DOI: 10.31526/ACP.NDM-2020.4

\section{INTRODUCTION}

Many astrophysical and cosmological observations indicate the existence of a significant contribution of non-baryonic dark matter (DM). However, the precise nature of dark matter beyond gravitational effects is still unknown. If DM is produced thermally in the early universe it can interact via the weak force with Standard Model particles and could therefore be detected still today. Dark matter self-annihilation is, for example, expected in locations of high dark matter densities. In addition, DM could be unstable with decay lifetimes larger than the age of the Universe. Indirect searches for dark matter therefore rely on the detection of an unexpected excess of secondary particles after DM annihilation or decay. Since neutrinos appear as final state particles in many possible processes, the IceCube neutrino observatory has an ideal opportunity to study or constrain the properties of DM particles.

With an instrumented volume of $1 \mathrm{~km}^{3}$, the IceCube neutrino observatory is the world's largest neutrino telescope [1]. It is located at the geographical South Pole at a depth between $1450 \mathrm{~m}$ and $2450 \mathrm{~m}$. Digital Optical Modules record the Cherencov light emitted by charged particles that are produced in the interactions of neutrinos in the surrounding ice or bedrock. Different event selections and reconstructions are used for different search strategies. Track events (mostly from $v_{\mu}$ charged current interactions) for example have a good angular resolution, which is ideal for point-like source regions such as the Sun, whereas cascade events with a better energy resolution make it possible to search for features in the energy spectrum and are used when studying the galactic halo and, for example, decaying dark matter where the directional information is less important. IceCube investigated a large variety of possible DM signals without any observation so far. In this contribution, we review a collection of recent results and give an outlook upon future developments.

\section{ANNIHILATION AND DECAY IN THE GALACTIC HALO}

The observable flux of neutrinos originating in dark matter annihilation or decay in the galactic DM halo strongly depends on the halo density $\rho_{\chi}$, which is the most important source of uncertainty. Therefore both a cuspy (i.e. peaked towards the galactic center, for example the NFW profile) and isothermal (e.g. Burkert) profiles are typically considered (see for example [2]). In the case of DM annihilation, the signal enhancement towards the direction of the galactic center is largest. Figure 1 shows a collection of limits set by IceCube data on the thermally averaged dark matter annihilation cross-section $\left\langle\sigma_{\mathrm{A}} v\right\rangle$ [3] (left) and DM decay lifetime [4] (right) in comparison with results from other experiments. Especially for high masses, the respective IceCube limits are leading constraints. At lower masses, the bounds from gamma-ray telescopes are still more stringent. One particular interesting scenario for IceCube is the direct annihilation or decay into neutrino pairs $\chi(+\chi) \rightarrow v+v$ where only few gamma rays are produced via electroweak bremsstrahlung. In addition, the resulting neutrinos have a distinct energy equal to (half the) dark matter mass in the case of (decay) annihilation. A new analysis is currently in preparation which exploits this fact by searching for a distinct line-like feature in the neutrino energy spectrum. The expected sensitivity of this new analysis is expected to significantly improve the existing limits up to masses of several TeV. In addition, new ways are explored to probe lower dark matter masses for example with updated event selections or with the upcoming low energy upgrade of IceCube [5].

${ }^{*}$ http://icecube.wisc.edu 

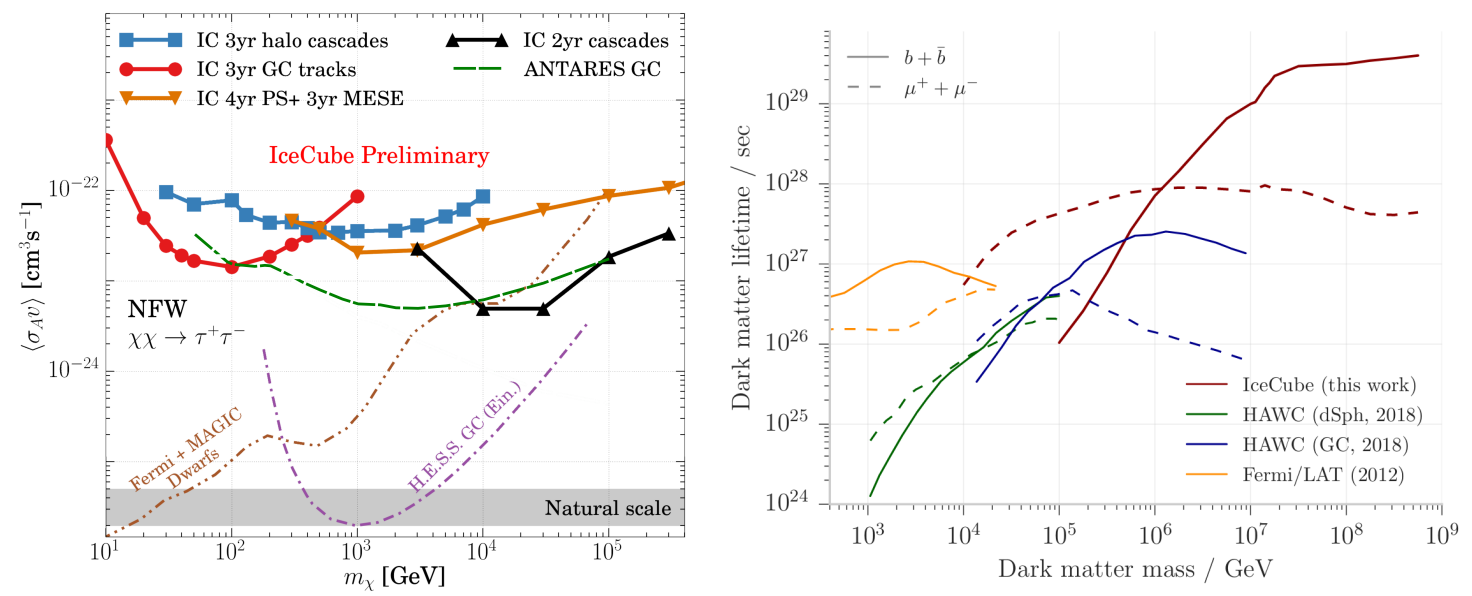

FIGURE 1: Left: Comparison of the upper limit on the thermally averaged dark matter annihilation cross-section obtained with different analyses using different event samples. Limits are given for a NFW dark matter halo profile and assuming annihilation into $\tau^{+} \tau^{-}$[3]. Right: Lower limits on the dark matter decay lifetime [4].
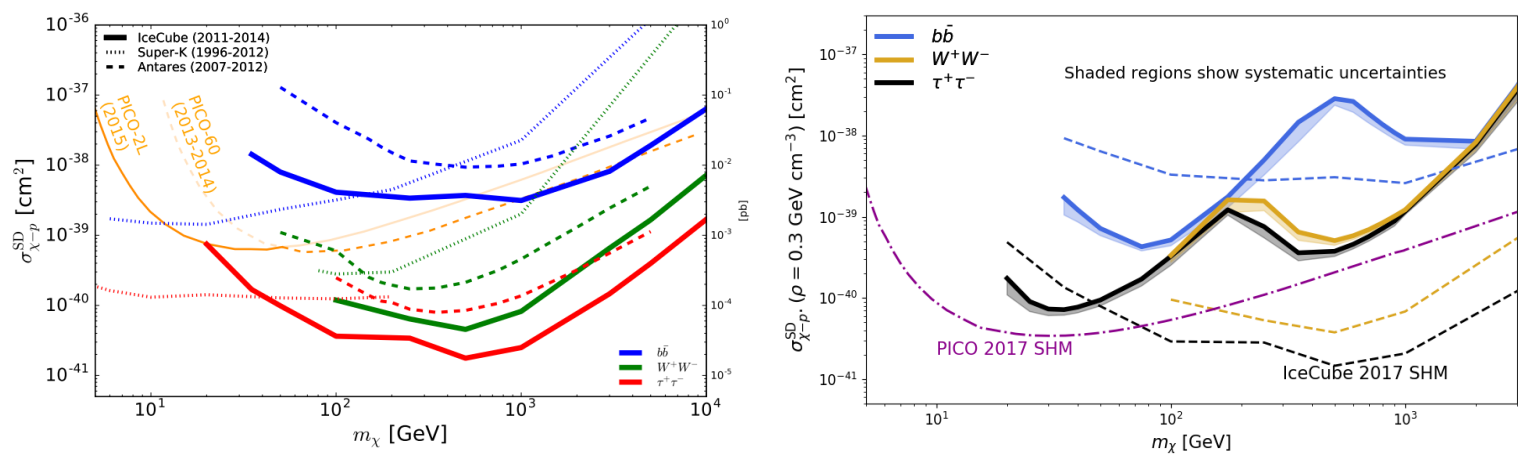

FIGURE 2: Left: Upper limits on the spin-dependent dark matter-proton cross-section $\sigma_{\chi-p}^{S D}$ compared to results from other neutrino detectors and direct detection experiments [6]. Right: Velocity independent limits obtained by the combination of IceCube and PICO data [7].

\section{ANNIHILATION IN THE CENTER OF THE SUN}

Assuming that DM particles weakly interact with Standard Model particles, they are likely to scatter off the nuclei in dense celestial bodies, loose momentum, and then get gravitationally captured. As a consequence, DM can accumulate in the core of the Sun, where self-annihilation is then enhanced. Due to their small interaction cross-section, neutrinos are the only standard model particles able to escape from the center of the Sun and therefore are promising messengers for dark matter annihilation. Given the age of the Sun and its mass, and equilibrium between capturing and annihilation can be assumed. Therefore, the flux of DM signal neutrinos from the Sun is proportional to the spin-dependent dark matter-proton scattering cross-section $\sigma_{\chi-p}^{S D}$. Further, the flux of neutrinos depends only on the local dark matter density, reducing the systematic uncertainties due to the dark matter halo profile. So far, no such excess of neutrinos from the direction of the Sun was observed and the corresponding upper limits on $\sigma_{\chi-p}^{S D}$ have been reported [6], see Fig. 2 (left)). The limits are most stringent at the TeV scale and exceed those of direct detection experiments. Direct and indirect detection methods, however, differ because of their different sensitivity to the velocity distribution of the local dark matter: the low velocity component is most important for the DM capture in the Sun while high velocities dominate the energy deposit in direct detection experiments. By combining the data of the IceCube experiment with those from PICO, it is therefore for the first time possible to derive upper limits on $\sigma_{\chi-p}^{S D}$ without any dependence on the underlying velocity distribution [7]. The obtained result is shown in the right panel of Fig. 2 and emphasizes the complementarity of direct and indirect searches.

In a similar way, dark matter can accumulate in the center of the Earth and the subsequent annihilation can lead to an excess of vertically upgoing neutrino events in the IceCube detector. A previous search with one year of data [8] is currently being updated and a significantly improved sensitivity on the spin-independent dark matter-nucleon cross-section is expected [9]. Preliminary sensitivities on the annihilation rate $\Gamma_{\mathrm{A}}$ are shown on the left panel of Fig. 3. 

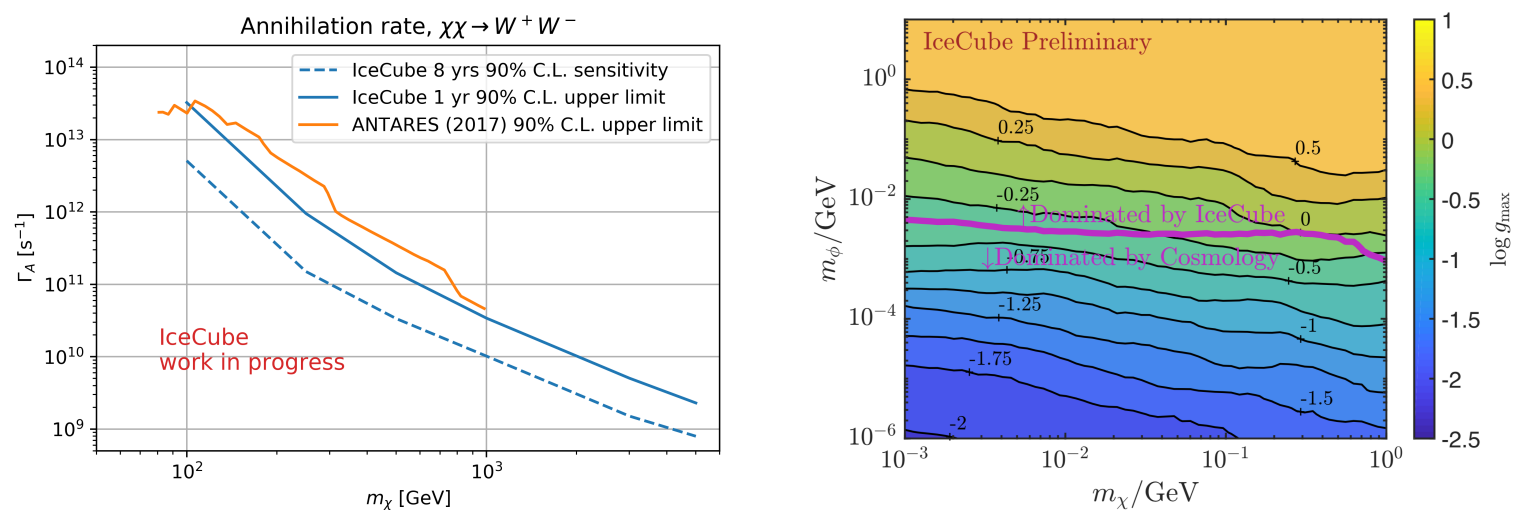

FIGURE 3: Left: Expected sensitivity on the dark matter annihilation rate in the center of the Earth for eight years of IceCube data [9]. Right: Upper limits on the dark matter - neutrino coupling as function of the dark matter mass and the mediator mass in the case of a dark matter fermion and mediator boson [10].

\section{NEUTRINO - DARK MATTER SCATTERING}

The high energy neutrino sample of IceCube is dominated by astrophysical neutrinos of most likely extra-galactic origin. Their directional distribution is compatible with isotropy. If DM couples to neutrinos, this directional distribution should be changed by an increased scattering of neutrinos traveling through the galactic dark matter halo. This deviation from isotropy is manifest by a suppressed neutrino flux from the direction of the galactic center. IceCube probed this hypothesis using high-energy starting events recorded in 7.5 years [10]. A simplified model is assumed in which DM is represented as a fermion (scalar) with a vector boson (fermion) mediator for the neutrino coupling. No flux suppression is found towards the Galactic Center and therefore limits on the dark matter-neutrino coupling are derived. The obtained limits of the coupling are shown in the right panel of Fig. 3 as a function of the DM and mediator mass for the fermion dark matter scenario. The derived limits are the most stringent bounds for mediator masses above $10 \mathrm{MeV}$ while bounds from cosmological observations are dominant for lower mediator masses.

\section{CONCLUSIONS}

The IceCube experiment has a lively program of indirect dark matter searches via neutrinos. The obtained limits and constraints on dark matter properties are complementary to other search strategies. Especially due to the fact that neutrinos can escape from the core of the Sun where dark matter would annihilate, IceCube is able to set the best limits on the spin-dependent dark matter-proton cross-section for some regions of the parameter space. IceCube is further improving the methods and strategies in order to improve the obtained constraints. Future detector upgrades will also enhance the telescopes reconstruction capabilities and sensitivity.

\section{References}

[1] M. G. Aartsen et al. The IceCube Neutrino Observatory: Instrumentation and Online Systems. JINST, 12(03):P03012, 2017.

[2] Fabrizio Nesti and Paolo Salucci. The Dark Matter halo of the Milky Way, AD 2013. JCAP, 1307:016, 2013.

[3] M. G. Aartsen et al. Search for Neutrinos from Dark Matter Self-Annihilations in the center of the Milky Way with 3 years of IceCube/DeepCore. Eur. Phys. J. C, 77(9):627, 2017.

[4] M. G. Aartsen et al. Search for neutrinos from decaying dark matter with IceCube. Eur. Phys. J., C78(10):831, 2018.

[5] Sebastian Baur. Dark matter searches with the IceCube Upgrade. PoS, ICRC2019:506, 2020.

[6] M. G. Aartsen et al. Search for annihilating dark matter in the Sun with 3 years of IceCube data. Eur. Phys. J., C77(3):146, 2017. [Erratum: Eur. Phys. J.C79,no.3,214(2019)].

[7] M. G. Aartsen et al. Velocity Independent Constraints on Spin-Dependent DM-Nucleon Interactions from IceCube and PICO. 2019.

[8] M.G. Aartsen et al. First search for dark matter annihilations in the Earth with the IceCube Detector. Eur. Phys. J. C, 77(2):82, 2017.

[9] Giovanni Renzi. Search for dark matter annihilation in the center of the Earth with 8 years of IceCube data. PoS, ICRC2019:541, 2020.

[10] Argüelles, Carlos A. and Dujmovic, Hrvoje. Searches for Connections Between Dark Matter and Neutrinos with the IceCube High-Energy Starting Event Sample. PoS, ICRC2019:839, 2020. 